\title{
Low-Frequency Component Electric Microfield Distributions in Plasmas*
}

C. F. Hooper, Jr.

Department of Physics \& Astronomy, University of Florida

Gainesville, Florida

\section{ABSTRACT}

Low-frequency component electric microfield distributions in a plasma are calculated at both a neutral and charged point. It is shown that this calculation allows for the inclusion of all correlations to a high degree of accuracy. The theory is compared with the Holtsmark and Baranger-Mozer theories. A detailed analysis of all approximations is included, together with a Monte Carlo study. Numerical results are shown both graphically and in tabulated form.

* This research was supported in part by the Research Corporation, New York, New York and the National Aeronautics and Space Administratinn

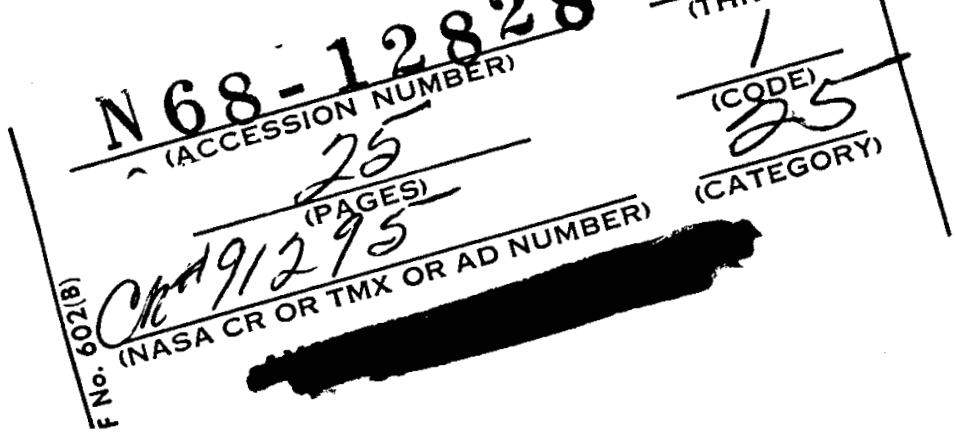




\section{TNTRODUCTION}

This is the second paper dealing with the problem of electric-microfield distributions in plasmas. The first, hereafter referred to as $I$, dealt with high-frequency component plasmas, where the plasma was assumed to consist of $\mathrm{N}$-charged particles moving in a uniform neutralizing background ${ }^{1}$. These $\mathrm{N}$ particles interacted with each other through a Coulomb potential.

The problem of the low-frequency component is the subject of this naper. Here the plasma is represented as a collection of $\mathrm{N}$ singly-charged shielded ions which interact with each other through an effective potential. The effective potential includes the effect of the ion-electron interactions. This model of a plasma has been, and is currently, used when dealing with the effects of ions on radiating atoms and/or ions immersed in a plasma 2,3 . The $N$ perturbing ions in the plasma are assumed to interact with each other through a shielded Coulomb or Debye-Hückel potential. The plasma is considered to be in thermal equilibrium and macroscopically neutral.

When treating the problem of the electric field distribution at a charged particle, an additional $(\mathrm{N}+1)$ st particle, conveniently placed at the origin of the reference frame, must be included. As in $I$, the problem of the electricmicrofield distribution at a neutral point is just a special case of the chargedpoint development.

Section II of this paper outlines the development of the formalism. The numerical results and analysis, including a comparison with the $B-M$ theory $^{4}$, are discussed in Section III. Final conclusions are presented in the fourth and final section. 
The equations presented in this section originate from the formalism developed in I. Again, the problem is the calculation of the electric-microfield distribution function, $P(\varepsilon)$, which is found from an evaluation of the equation

$$
P(\varepsilon)=2 \pi^{-1} \varepsilon \int_{0}^{\infty} T(\ell) \sin (\varepsilon \ell) \ell d \ell,
$$

where $T(l)$ is given by,

$$
T(l)=z^{-1} \int \cdots \int \exp \left[-\beta V+i \sum_{j} \ell \cdot\left(\frac{e^{r} j}{r_{j}^{3}}\left[1+\frac{r_{j}}{\lambda}\right] e^{r_{j} / \lambda}\right)\right] \pi_{j} d f_{j},
$$

where

$$
V=\sum_{0=i<j}^{N} \frac{e^{2}}{r_{i j}} e^{-r_{i j} / \lambda}
$$

and $\lambda$ is the Debye length,

$$
\lambda=\left(k T / 4 \pi n e^{2}\right)^{1 / 2}
$$

Now express the total potential, $V$, in the form

$$
V=v_{0}+\sum_{i} w_{i o}
$$

where

$$
w_{\text {io }}=\frac{e^{2}}{r_{\text {io }}} e^{-\alpha r_{i o} / \lambda}
$$

$\alpha$ is an arbitrary positive constant.

With these basic definitions we can follow the procedure in I (Eq.12 to 24 ) to arrive at the following expression for $T(l)$ : 


$$
T(l)=\left[T_{0}(l) / T_{0}(0)\right] \exp \left\{\sum_{j}\left(n^{j} / j !\right)\left[h_{j}(l)-h_{j}(0)\right]\right\} .
$$

Again as in $I$, the factor $T_{0}(l) / T_{0}(0)$ has the form

$$
\left[T_{0}(l) / T_{0}(0)\right]=e^{-\gamma L^{2}}
$$

where $\gamma$ is given by the expression:

$$
\gamma=\left[a / 4\left(\alpha^{2}-2\right)^{2}\right]\left[\alpha^{5}+2(1-2 \sqrt{2}) \alpha^{4}+3 \alpha^{3}+8(\sqrt{2}-1) \alpha^{2}-6 \alpha+4(2-\sqrt{2})\right],
$$

and where

$$
L \equiv l \varepsilon_{0}, \quad a \equiv r_{0} / \lambda, \quad \varepsilon_{0} \equiv \mathrm{e} / \mathrm{r}_{0}^{2}
$$

$r_{0}$ is the much discussed ion-sphere radius which is defined by the relation

$$
\frac{4}{3} \pi r_{0}^{3} n=1
$$

Next, we consider the factors resulting from the terms in the series exponent. For $j=1$, we find, using Eqs. 28 and 29 of $I$, that

$$
\begin{gathered}
I_{1}=n\left[h_{1}(l)-h_{1}(0)\right] \\
I_{1}=3 \int_{0}^{\infty} d x x^{2} e^{F(x)}\left[\frac{\sin [L G(x)]}{[L G(x)]}-I\right]-3 \int_{0}^{\infty} d x x^{2} e^{s(x)}\left[\frac{\sin [L q(x)]}{[L q(x)]}-1\right]
\end{gathered}
$$

where:

$$
\begin{gathered}
x \equiv r / x_{0} \\
F(x)=\frac{a^{2}}{3 x}\left[\frac{1}{\alpha^{2}-2}\right]\left[e^{-\alpha a x}-\left(\alpha^{2}-1\right) e^{-\sqrt{2} a x}\right] \\
G(x)=\left(\alpha^{2}-2\right)^{-1}\left[\frac{1}{x}\left[\left(\alpha^{2}-1\right) e^{-\sqrt{2 a x}}-e^{-\alpha a x}\right]\right.
\end{gathered}
$$




$$
\begin{gathered}
\left.+\frac{a}{x}\left[\sqrt{2}\left(\alpha^{2}-1\right) e^{-\sqrt{2} a x}-\alpha e^{-\alpha a x}\right]\right] \\
s(x)=\frac{a^{2}}{3 x}\left[\frac{\alpha^{2}-1}{\alpha^{2}-2}\right]\left\{e^{-\alpha a x}-e^{-\sqrt{2} a x}\right\} \\
q(x)=\left[\frac{\alpha^{2}-1}{\alpha^{2}-2}\right]\left[\frac{1}{x^{2}}\left(e^{-\sqrt{2} a x}-e^{-\alpha a x}\right)-\frac{a}{x}\left(\alpha e^{-\alpha a x}-\sqrt{2} e^{-\sqrt{2} a x}\right)\right]
\end{gathered}
$$

Following Eqs.31 to 33 in I, we find for the second term in the series exponent $(j=2)$ the expression:

$$
\begin{aligned}
& I_{2}(l)=\frac{1}{2} n^{2}\left[h_{2}(l)-h_{2}(0)\right] \\
& =\sum_{k}(-1)^{k+1} 3(2 k+1) a^{2}\{\} \\
& \{\}=\left\{\int _ { 0 } ^ { \infty } e ^ { s ( x _ { 2 } ) } I _ { k + 1 / 2 } ( a ^ { \prime } x _ { 2 } ) \left[e^{-\beta w_{2} 0} j_{k}\left[L G\left(x_{2}\right)\right]-j_{k}\left[\left(L q\left(x_{2}\right)\right]\right] x_{2}{ }^{3 / 2}\right.\right. \\
& \times\left[\int_{x_{2}}^{\infty} e^{s\left(x_{1}\right)} K_{k+1 / 2}\left(a^{\prime} x_{1}\right)\left[e^{-\beta w_{1} 0} j_{k}\left[L G\left(x_{1}\right)\right]-j_{k}\left[L q\left(x_{1}\right)\right] x_{1}{ }^{3 / 2} d x_{1}\right] d x_{2}\right. \\
& \left.-\delta_{k o} \int_{0}^{\infty} e^{s\left(x_{2}\right)} I_{1 / 2}\left(a^{\prime} x_{2}\right)\left[e^{-\beta w} 20-1\right] x_{2}^{3 / 2}\left[\int_{x_{2}}^{\infty} e^{s\left(x_{1}\right)} k_{1 / 2}\left(a^{\prime} x_{1}\right)\left[e^{-\beta w} 10-1\right] x_{1}^{3 / 2} d x_{1}\right] d x_{2}\right\}
\end{aligned}
$$

Four of the functions appearing in the above equation, $G(x), w_{10}, q(x)$, and $s(x)$ have already been defined. It remains to note that the $j_{k}(-)$ represents a spherical Bessel function of order $k$, while $I$ and $K$ refer to modified Bessel functions of the first and third kind respectively. 5 Another feature of this last equation which should be noted is that the argument of the modified Bessel function involves an $a^{\prime}$ which is defined by the relation $a^{\prime}=\sqrt{2 a}$. Thus we finally arrive at the result,

$$
T(\ell)=\exp \left[-\gamma^{2}+I_{1}(\ell)+I_{2}(\ell)\right]
$$

This expression is used in Eq.(1) to calculate $P(\varepsilon)$ at a charged particle. 



$$
-7-
$$

over collective coordinates which have a form that is easily evaluated. For convenience, the form of these collective-coordinate integrals, along with solutions is stated below:

$$
\begin{aligned}
& I=\int \cdots \int \exp \left\{-\frac{l}{2} \sum_{k}\left[A_{k} x_{k}{ }^{2}+2 b_{k} x_{k}\right]\right\} J_{k} d x_{k} \\
& =\text { const } \times \exp \left\{\frac{1}{2}\left[{ }_{k} b_{k}{ }^{2 /\left(1+A_{k}\right)}\right\} \times\left[1-a_{3}+a_{4} \cdots\right]\right. \text {, }
\end{aligned}
$$

where $A_{k}$ and $b_{k}$ are specific functions of $k$, the $x_{k}$ 's represent collective coordinates, and $J$ is the Jacobian of the $\underset{\sim}{r} \rightarrow \underset{\sim}{X}$ transformation. The series of terms in brackets represents the possible higher order corrections to the first Jacobian approximation. In the calculations made thus far, $\mathbf{a}_{3}, \mathbf{a}_{4}$, etc. have been neglected. We assume, of course, that these correction terms are negligible. However, this assertion must be verified.

The first step in using, and in evaluating the present theory, is to determine the adjustable parameter $\alpha$. Perhaps the best choice of $\alpha$ is the one which results in a minimum error due to the combined effect of the cluster-expansion termination error and the Jacobian error. An even better choice of $\alpha$ would. be one which resulted in the error due to each of the two major sources being negligible, if this is possible. A clear indication that such a circumstance had occurred would be the existence of a distinct and extended range of $\alpha$ values over which the $T(\ell)$ curve, and hence the $P(\varepsilon)$ curve, would remain stationary; the requirement of such a range would virtually rule out any possibility that the two errors had merely cancelled one another. The latter choice was shown to be possible, and was the one chosen to determine the best value of $\alpha$; specifically, an $\alpha$ value lying at the approximate center of the stationary range was the one chosen. 
Rather than rely solely on the above argument, this criterion was subjected to several tests. First, the second term in the cluster expansion was calculated, and is shown in Fig. 1 to contribute less than $2 \%$ to any point on the $P(\varepsilon)$ curve in a "worst case" situation. Similarly, the $a_{3}$ term in the Jacobian correction series was evaluated. It is shown in Fig.2 that, in the a region chosen for the calculation, this term, which indicates skewness of the collective coordinate distribution, is negligible. From the related structure of $a_{4}$ it may be deduced that the same choice of $\alpha$ will also make $a_{4}$ negligible. In an effort to also rule out the possibility that although $a_{3}$ and $a_{4}$ are small, the entire series is appreciable, a special case is considered.

Since the theory of $B-M$ should indeed be valid for dilute systems at sufficiently high temperature, $P(\varepsilon)$ curves predicted by both theories at $\underline{a}=$ 0.2 , should agree quite well ( $\underline{a}=0.0$ corresponds to the Holtsmark case). It is shown graphically in the next section that, in this instance, the present theory without Jacobian corrections yields a $P(\varepsilon)$ curve almost identical to that predicted from the B-M theory. The assertion is that in this case, $a_{3}, a_{4}$, and the entire Jacobian correction series are really negligible. Figure 2 indicates that freedom to choose the correct $\alpha$ value corresponding to a given $\underline{a}$ results in $a_{3}$ having at least the same order of magnitude for $\underline{a}>0.2$ as it did when $\underline{a}=0.2$. If the $\alpha$ variation affects $a_{3}$ and $a_{4}$ in this manner, it is plausible to expect the entire series to be similarly affected. Thus, by this argument too, we expect the present theory without Jacobian correction to be valid for rather high-density, low-temperature regions (e.g., $\underline{a}=0.8)$.

A final attempt at verifying the procedure is shown in Fig.3. Here we see a comparison of a Monte Carlo calculation of $P(\varepsilon)$ for $a=0.8$ (the largest 


$$
-9-
$$

deviation from the Holtsnark distribution considered in this paper) and the corresponding curve for this theory. It is seen that although there are still some fluctuations in the Monte Carlo curve, it agrees quite well with the result predicted by the present theory and would seem to further substantiate the present result.

Actual numerical results are discussed in detail in the next section.

III. Numerical Results and Analysis

It should again be emphasized that all studies of the $\alpha$ flatness region indicate that in this range the disregarded corrections due to both major sources of error are indeed negligible. Figures 1 and 2 show these results. While Fig.l is self-explanatory, Fig. 2 may be understood as follows: We may write 6

$$
a_{3}=a_{3}{ }^{0}-\gamma^{\prime} L^{2}
$$

Since $a_{3}{ }^{0}$ is not a function of $\underline{\ell}$ and since, in addition, it is very small compared to unity $\left(\simeq 10^{-5}\right)$ for all cases considered, it is set equal to zero' in all further discussions. In order to gain some impression of the importance of the $a_{3}$ correction, we consider its influence on the calculated values of $T_{0}(l) / T_{0}(0)$. In view of the fact that $\gamma^{\prime}$ is quite smal1, it is permissible to write

$$
\begin{aligned}
T_{0}(l) T_{0}(0) & \simeq \exp \left\{-\gamma L^{2}\right\}\left[1+\gamma^{\prime} L^{2}\right] \\
& \simeq \exp \left\{-\left(\gamma-\gamma^{\prime}\right) L^{2}\right\}
\end{aligned}
$$

A measure of the importance of the correction due to $a_{3}$ in this instance may be given by plotting the ratio of $\gamma^{\prime} / \gamma$ versus $\alpha$ for a values of interest. 
It is clearly seen from Fig. 2 that in the regions of flatness, $a_{3}$ amounts to an insignificant correction.

Figures 4 and 5 show graphs of $P(\varepsilon)$ versus $\varepsilon$ for several values of $\alpha$, while Figs. 6 and 7 indicate the differences occurring between the B-M theory and the present theory for cases characterized by $\underline{a}=0.2$ and $\underline{a}=0.8$. It will be noticed that the difference between the two theories increases as the magnitude of a increases, and that the B-M theory favors weaker fields than does the proposed theory. One possible explanation for the direction of the difference between the two may lie in the fact that in Baranger's second correction term to $T(l), \exp \left[L^{3 / 2} \Psi_{2}\left(a L^{1 / 2}\right)\right]$, the linearized pair-correlation function is used instead of the non-1inearized form. It has been argued by $B-M$ that the difference between the two functional forms should not really matter since the procedure was "also in the spirit of the Debye-Hückel theory."7 However, Fig. 8 illustrates that the effect of a reduction in $\Psi_{2}\left(a L^{1 / 2}\right)$ on the final $P(\varepsilon)$ curve may be very large. A similar reduction in the second correction term in the present theory leads to only a slight change in the $P(\varepsilon)$ curve under identical conditions; this may be deduced from Fig.1. A reduction in the magnitude of $\Psi_{2}$ is what one would expect if the nonlinearized DebyeHuckel function were used instead of the linearized version: this is because the linearized form underestimates the contribution to the pair-correlation function from strong fields and hence overemphasizes the $\Psi_{2}$ term.. It would be necessary to carry out a calculation of $\Psi_{2}$ using the nonlinearized function before the final magnitude of the reduction could be ascertained.

Tables I and II list some tabulated values of $P(\varepsilon)$ for reference. 


\section{Conclusion}

This paper discusses the calculation of electric microfields in lowfrequency component plasmas. It depends largely on I for the development of the necessary formalism. For the plasma under consideration, this method of calculating electric microfields in plasma has been shown to be effective over a wide temperature-density range; it goes to the Holtsmark 1imit as $\mathrm{T} \rightarrow \infty$, and at $\underline{a}=0.8$ it has been shown to predict a reliable result. A comparison of this method with that of B-M clearly indicates that while the latter is good at high temperatures and low densities (a $=0.2$ ) it becomes progressively inaccurate as $\underline{a}$ is raised from 0.2 to 0.8 . The net result of these calculations is that for the charged-point and the nuetral-point cases, the distribution curves generated by the present theory favor slightly stronger fields than does the theory of $B-M$.

As in $I$, the method of including noncentral forces through the mechanism of collective coordinates is shown to be highly effective. Exactly how good this method is, is evidenced when the second term in the cluster expansion is calculated; here one finds that the noncentral, two-particle correlations are included, through the use of collective coordinates, to the approximation of the nonlinear Debye-Hückel result. Since this second term is only a small correction to the theory, even in the case of high a values, such an approximation must be considered highly accurate. Furthermore, during the derivation of the general formalism, especially that part relating to the cluster expansion, the fact that it was not necessary to explicitly mention noncentral interactions resulted in much simplification. 
FOOTNOTES

1. C. F. Hooper, Jr., Phys. Rev. 149, 77 (1966).

2. H. R. Griem, M. Baranger, A. C. Kolb, and G. Dertel, Phys. Rev. 125, 177 (1962).

3. Earl W. Smith and C. F. Hooper, Jr., Phys. Rev. 157126 (1967).

4. B. Mozer and M. Baranger, Phys. Rev. 118, 626 (1960).

5. The Bateman Manuscript Project, Higher Transcendental Functions (California Institute of Technology, Pasadena, California, 1953) Vol.II, Chap. VII.

6. A. A. Broyles, Z. Physik 151, 187 (1958).

7. B. Mozer, Dissertation, Department of Physics, Carnegie Institute of Technology, 1960 (unpublished). 
FIGURE CAPTIONS

Fig. 1. A comparison of two $T(l)$ approximations as applied to the present theory of the electric microfield distribution function $P(\varepsilon)$ (Charged point case). $\varepsilon$ is in units of $\varepsilon_{0}$.

Fig. 2. An estimate of the importance of the Jacobian correction term, $a_{3}$. $P(\varepsilon)$ curves calculated for any of the $\alpha$ values lying in the heavy section of a given line will agree to within several percent (at worst) over the entire range of $\varepsilon$ values considered in this paper. For further explanation, see Section III of the text.

Fig. 3. A comparison of the electric microfield distribution $P(\varepsilon)$ calculated by the present theory, with that predicted by a Monte Carlo calculation (40,000 particle configurations).

Fig. 4. The electric microfield distribution function $P(\varepsilon)$, at a charged point, for several values of $\underline{a}$; $\varepsilon$ is in units of $\varepsilon_{0}$.

Fig. 5. The electric microfield distribution function $P(\varepsilon)$, at a neutral point, for several values of $a$; $\varepsilon$ is in units of $\varepsilon_{0}$.

Fig. 6. A comparison of the electric microfield distribution function (at a charged point) determined by $B-M$, with that predicted by the present theory; $\varepsilon$ is in units of $\varepsilon_{0}$.

Fig. 7. A comparison of the electric microfield distribution function (at a neutral point) determined by $B-M$, with that predicted by the present theory; $\varepsilon$ is in units of $\varepsilon_{0}$.

Fig. 8. A comparison of two $T(l)$ approximations as applied to the $B-M$ theory of the electric microfield distribution function $P(\varepsilon)$ (charged point case). $\varepsilon$ is in units of $\varepsilon_{0} \cdot$ 
Table I

Probability distributions, $P(\varepsilon)$, at a charged point for several values of a. The electric field strength, $\varepsilon$, is in units of $\varepsilon_{0}$.

\begin{tabular}{|c|c|c|c|c|}
\hline$\varepsilon$ & $a=0.2$ & $a=0.4$ & $a=0.6$ & $a=0.8$ \\
\hline 0.1 & 0.00710 & 0.01244 & 0.02229 & 0.04114 \\
\hline 0.2 & 0.02779 & 0.04801 & 0.08397 & 0.14866 \\
\hline 0.3 & 0.06028 & 0.10180 & 0.17146 & 0.28527 \\
\hline 0.4 & 0.10187 & 0.16687 & 0.26777 & 0.41354 \\
\hline 0.5 & 0.14926 & 0.23557 & 0.35752 & 0.51032 \\
\hline 0.6 & 0.19894 & 0.30091 & 0.43017 & 0.56853 \\
\hline 0.7 & 0.24755 & 0.35745 & 0.48081 & 0.59163 \\
\hline 0.8 & 0.29220 & 0.40179 & 0.50909 & 0.58755 \\
\hline 0.9 & 0.33065 & 0.43248 & 0.51766 & 0.56481 \\
\hline 1.0 & 0.36144 & 0.44972 & 0.51053 & 0.53069 \\
\hline 1.1 & 0.38385 & 0.45484 & 0.49199 & 0.49072 \\
\hline 1.2 & 0.39782 & 0.44982 & 0.46591 & 0.44876 \\
\hline 1.3 & 0.40385 & 0.43690 & 0.43546 & 0.40731 \\
\hline 1.4 & 0.40279 & 0.41822 & 0.40305 & 0.36786 \\
\hline 1.5 & 0.39573 & 0.39572 & 0.37041 & 0.33125 \\
\hline 1.6 & 0.38385 & 0.37102 & 0.33870 & 0.29782 \\
\hline 1.7 & 0.36832 & 0.34537 & 0.30864 & 0.26763 \\
\hline 1.8 & 0.35021 & 0.31974 & 0.28065 & 0.24057 \\
\hline 1.9 & 0.33049 & 0.29480 & 0.25489 & 0.21644 \\
\hline 2.0 & 0.30994 & 0.27100 & 0.23140 & 0.19498 \\
\hline 2.5 & 0.21235 & 0.17489 & 0.14420 & 0.11889 \\
\hline
\end{tabular}


Table I Cont'd.

$\varepsilon$
$a=0.2$
$a=0.4$
$a=0.6$
$a=0.8$

$\begin{array}{lllll}3.0 & 0.14096 & 0.11423 & 0.09330 & 0.07636 \\ 3.5 & 0.09496 & 0.07722 & 0.06310 & 0.05152 \\ 4.0 & 0.06601 & 0.05423 & 0.04447 & 0.03627 \\ 4.5 & 0.04749 & 0.03945 & 0.03247 & 0.02645 \\ 5.0 & 0.03528 & 0.02960 & 0.02443 & 0.01988 \\ 6.0 & 0.02112 & 0.01798 & 0.01489 & 0.01205 \\ 7.0 & 0.01375 & 0.01181 & 0.00978 & 0.00788 \\ 8.0 & 0.00955 & 0.00823 & 0.00680 & 0.00544 \\ 9.0 & 0.00697 & 0.00604 & 0.00499 & 0.00399 \\ 10.0 & 0.00526 & 0.00455 & 0.00373 & 0.00297\end{array}$


Table II

Probability distributions, $P(\varepsilon)$, at a Neutral point for several values of $\underline{a}$. The electric field strength, $\varepsilon$, is in units of $\varepsilon_{0}$.

\begin{tabular}{|c|c|c|c|c|}
\hline$\varepsilon$ & $a=0.2$ & $a=0.4$ & $a=0.6$. & $a=0.8$ \\
\hline 0.1 & 0.00696 & 0.01159 & 0.01938 & 0.03341 \\
\hline 0.2 & 0.02723 & 0.04475 & 0.07321 & 0.12143 \\
\hline 0.3 & 0.05908 & 0.09505 & 0.15020 & 0.23520 \\
\hline 0.4 & 0.09989 & 0.15612 & 0.23601 & 0.34511 \\
\hline 0.5 & 0.14643 & 0.22098 & 0.31745 & 0.43193 \\
\hline 0.6 & 0.19529 & 0.28313 & 0.38516 & 0.48866 \\
\hline 0.7 & 0.24319 & 0.33751 & 0.43440 & 0.51674 \\
\hline 0.8 & 0.28729 & 0.38083 & 0.46434 & 0.52160 \\
\hline 0.9 & 0.32539 & 0.41160 & 0.47677 & 0.50962 \\
\hline 1.0 & 0.35604 & 0.42985 & 0.47487 & 0.48656 \\
\hline 1.1 & 0.37851 & 0.43668 & 0.46215 & 0.45700 \\
\hline 1.2 & 0.39273 & 0.43384 & 0.44193 & 0.42432 \\
\hline 1.3 & 0.39914 & 0.42331 & 0.41706 & 0.39083 \\
\hline 1.4 & 0.39857 & 0.40709 & 0.38960 & 0.35803 \\
\hline 1.5 & 0.39206 & 0.38697 & 0.36132 & 0.32684 \\
\hline 1.6 & 0.38077 & 0.36447 & 0.33331 & 0.29775 \\
\hline 1.7 & 0.36582 & 0.34080 & 0.30633 & 0.27098 \\
\hline 1.8 & 0.34828 & 0.31689 & 0.28085 & 0.24658 \\
\hline 1.9 & 0.32908 & 0.29342 & 0.25710 & 0.22446 \\
\hline
\end{tabular}


Table II Cont'd.

\begin{tabular}{|c|c|c|c|c|}
\hline$\varepsilon$ & $a=0.2$ & $a=0.4$ & $a=0.6$ & $a=0.8$ \\
\hline 2.0 & 0.30900 & 0.27085 & 0.23520 & 0.20451 \\
\hline 2.5 & 0.21296 & 0.17812 & 0.15167 & 0.13120 \\
\hline 3.0 & 0.14208 & 0.11818 & 0.10098 & 0.08797 \\
\hline 3.5 & 0.09611 & 0.08095 & 0.06999 & 0.06162 \\
\hline 4.0 & 0.06704 & 0.05749 & 0.05038 & 0.04484 \\
\hline 4.5 & 0.04838 & 0.04224 & 0.03749 & 0.03371 \\
\hline 5.0 & 0.03603 & 0.03197 & 0.02870 & 0.02603 \\
\hline 6.0 & 0.02166 & 0.01971 & 0.01804 & 0.01659 \\
\hline 7.0 & 0.01415 & 0.01312 & 0.01217 & 0.01135 \\
\hline 8.0 & 0.09856 & 0.00925 & 0.00868 & 0.00815 \\
\hline 9.0 & 0.00722 & 0.00686 & 0.00652 & 0.00623 \\
\hline 10.0 & 0.00546 & 0.00522 & 0.00498 & 0.00480 \\
\hline
\end{tabular}




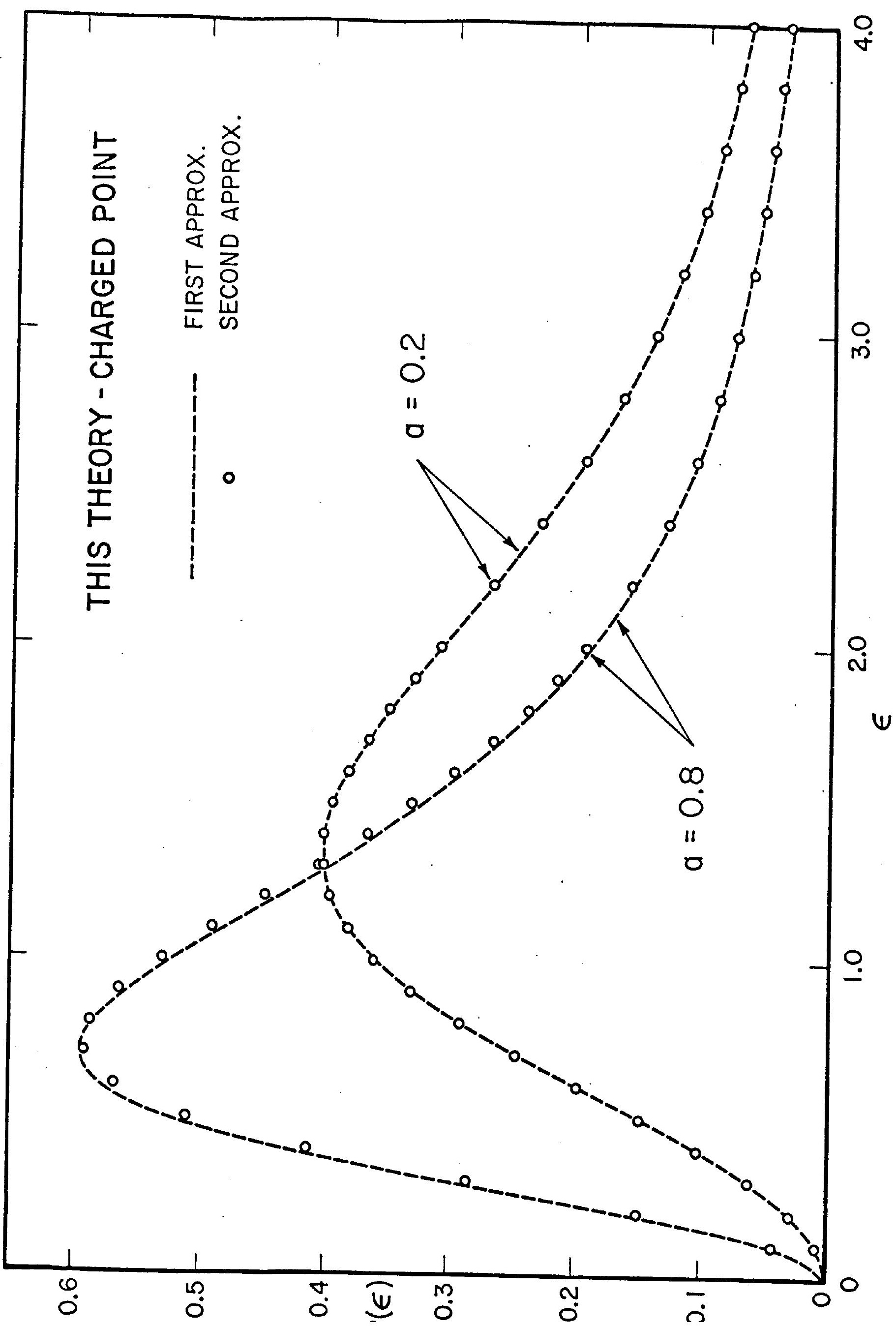




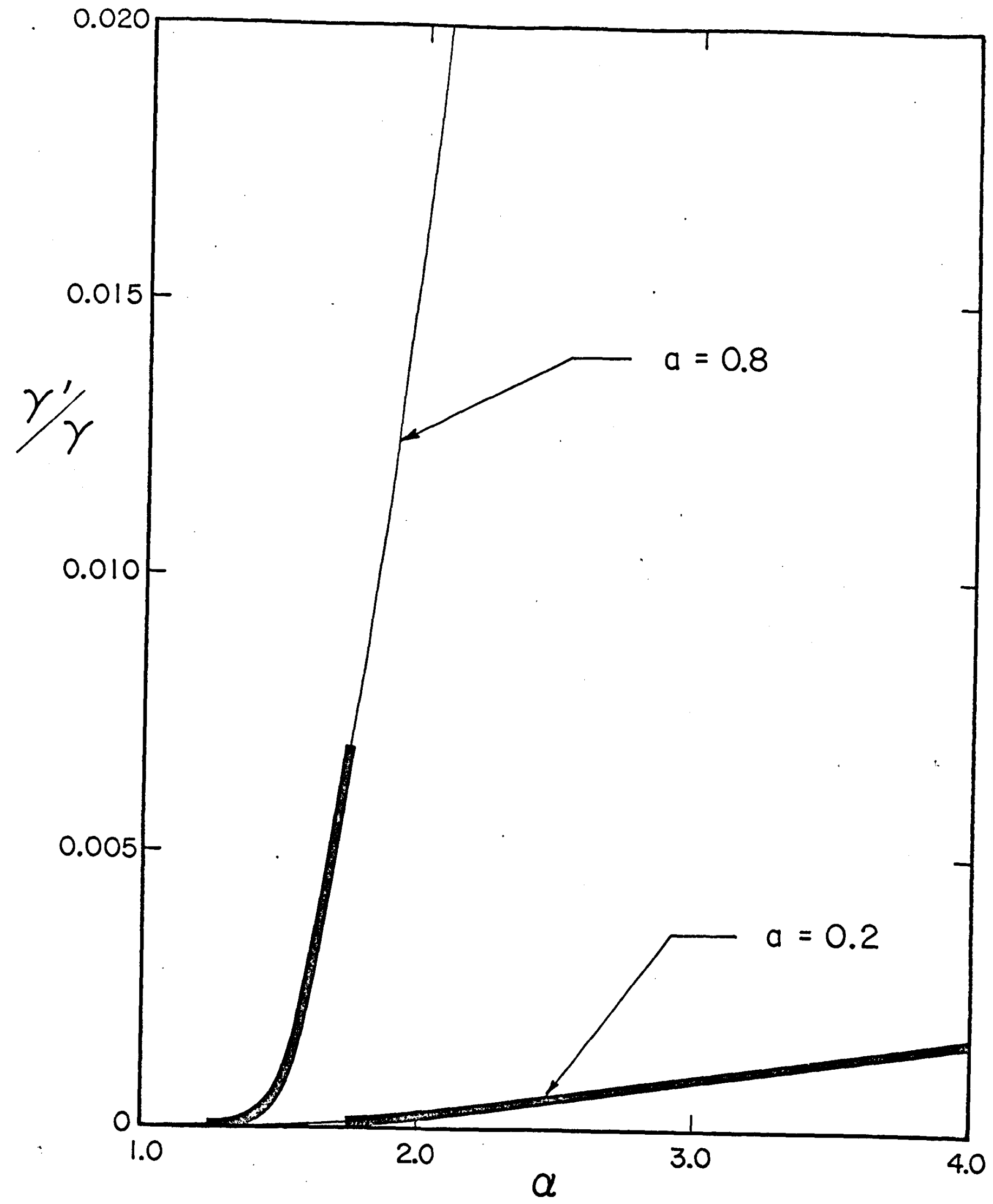




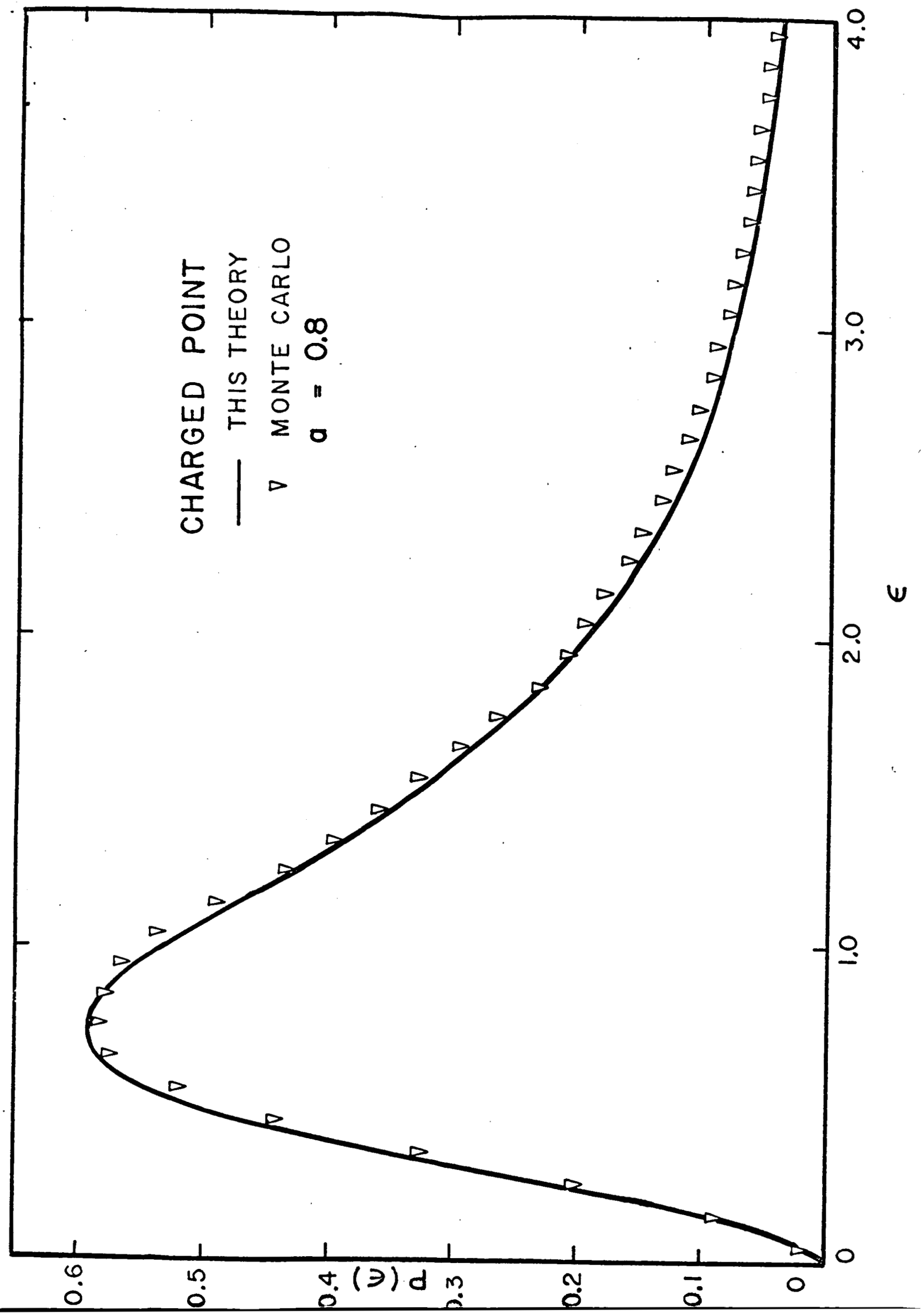




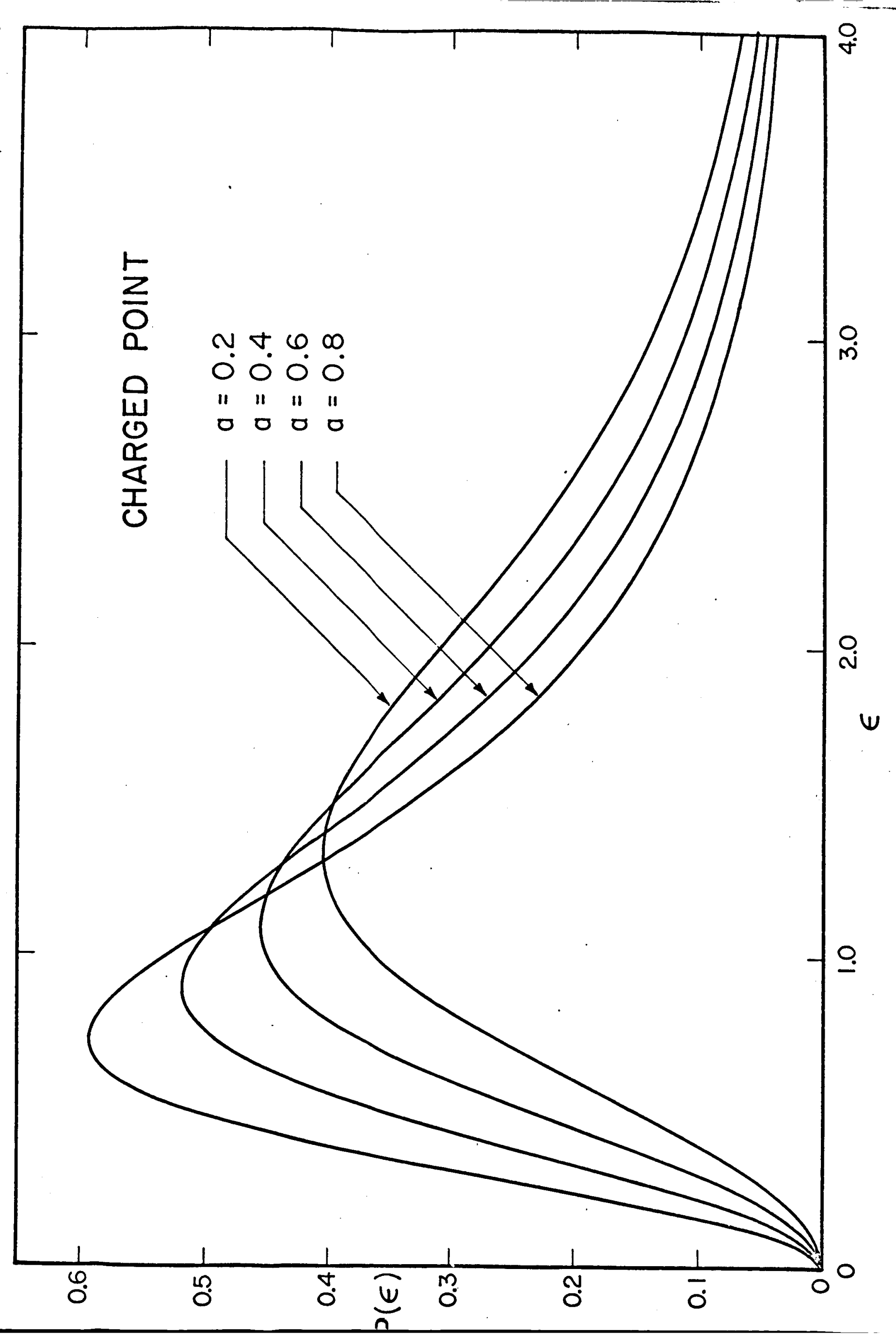




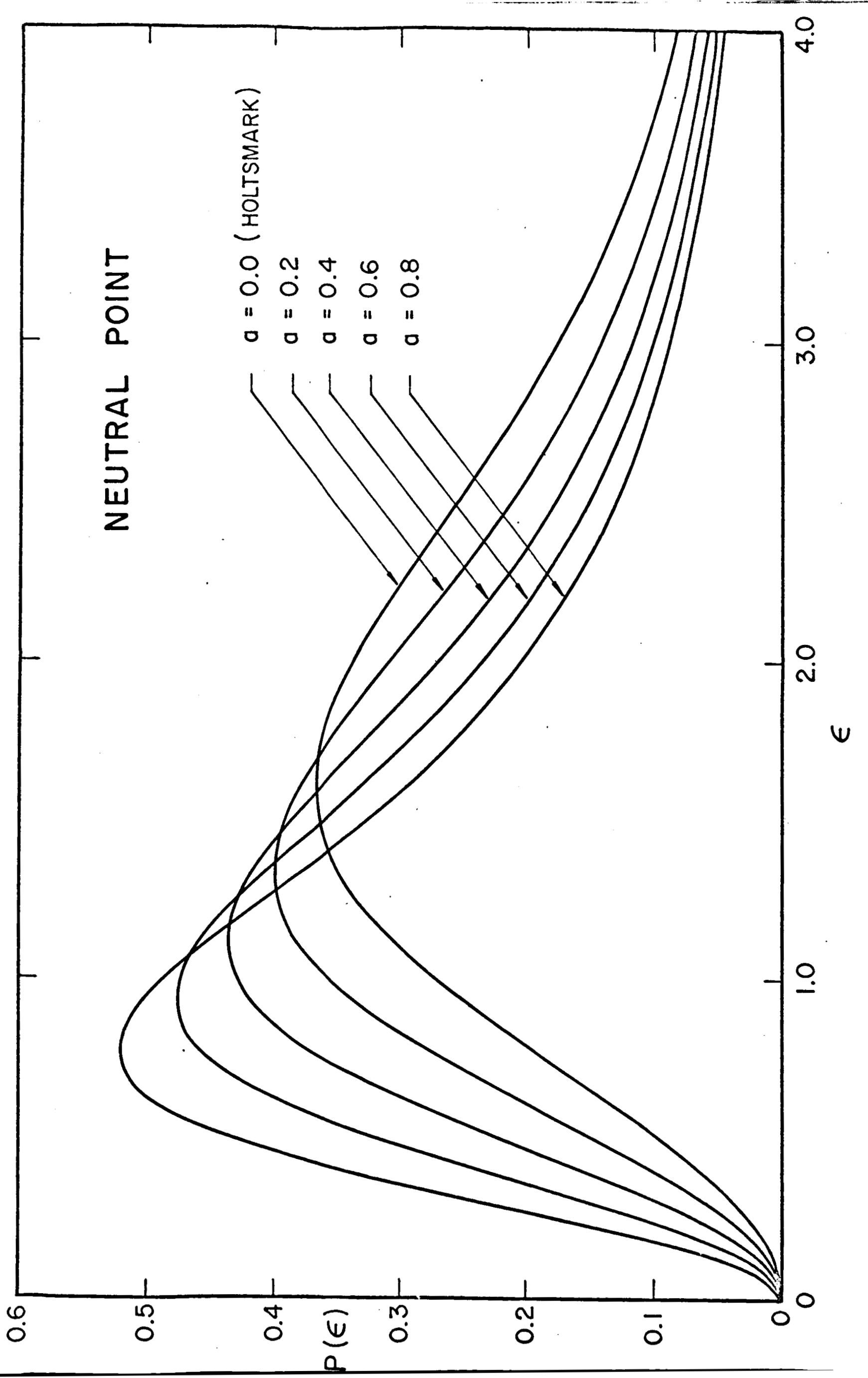




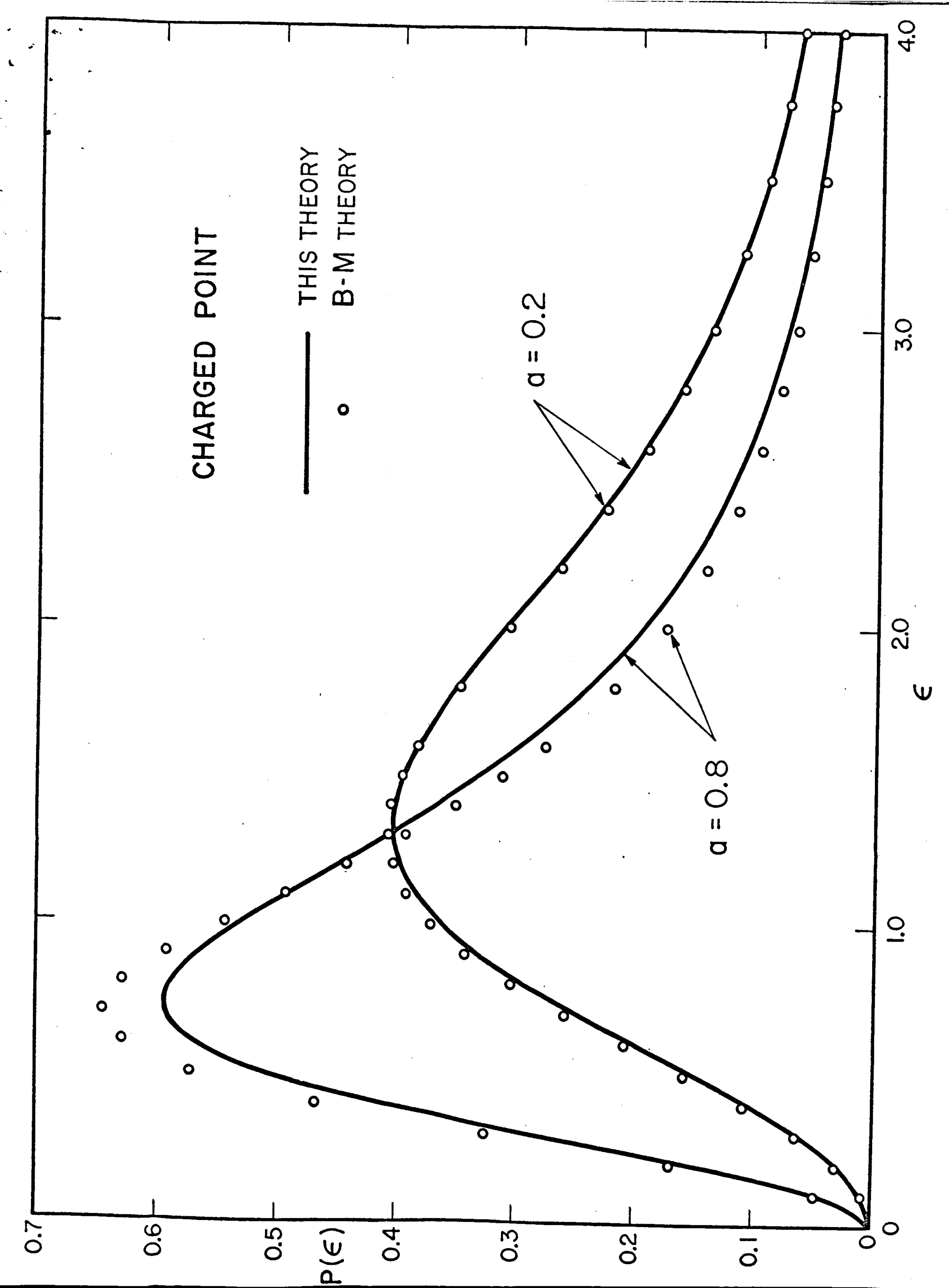




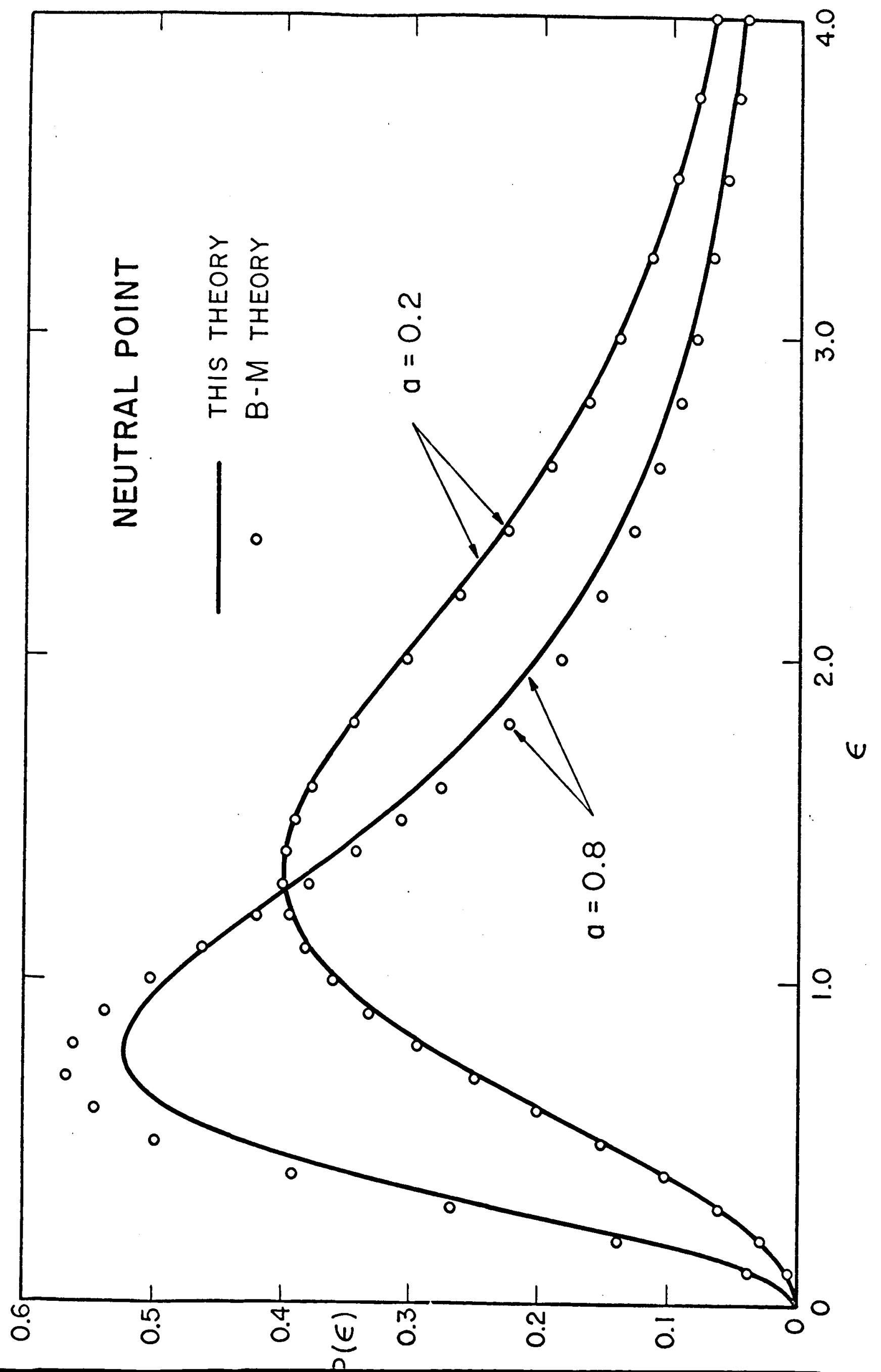




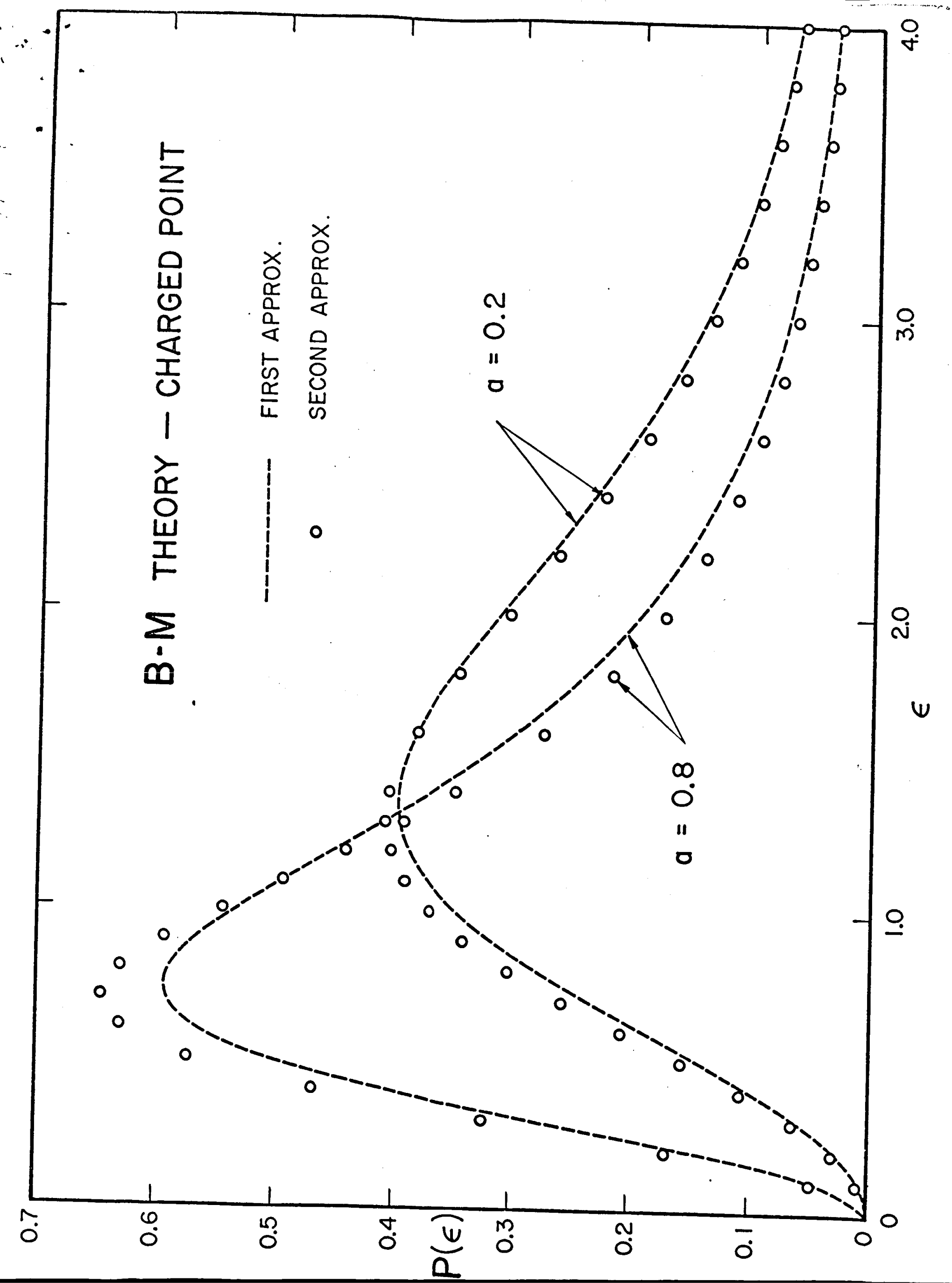

